\title{
Utilidad de la ecografía cardiaca a pie de cama en el manejo del taponamiento cardiaco
}

Víctor Pérez Cateriano ${ }^{1,2, a}$, Jamille Charlot Pasco Ulloa ${ }^{2, a}$

RESUMEN

En los últimos años, tanto en diferentes estudios como en la práctica clínica habitual, ha quedado demostrada la utilidad de la ecografía a pie de cama para optimizar el manejo de los pacientes críticos, debiendo ser hoy en día una herramienta más que nos guíe en el manejo de este tipo de pacientes. En este artículo se presenta la utilidad de la ecografía a pie de cama en la detección y manejo de una paciente con signos iniciales de taponamiento cardiaco, antes de que presente hipotensión severa, lo que permitió tomar una decisión pronta para su manejo y condicionó posteriormente su recuperación.

Palabras clave: Ecografía; pie de cama; taponamiento cardiaco (Fuente: DeSC BIREME).

\section{Utility of bedside cardiac ultrasound in the management of cardiac tamponade}

\begin{abstract}
In recent years, both in different studies and in usual clinical practice, it has been demonstrated the utility of bedside ultrasound to optimize the management of critically ill patients. Today it should be an additional tool to guide us in the management of these patients. This article presents the utility of bedside ultrasound in the disease detection and management of a female patient with early signs of cardiac tamponade before the development of severe hypotension. Such ultrasound allowed making an early decision for her treatment, which subsequently affected her recovery.
\end{abstract}

Keywords: Ultrasound; bedside; cardiac tamponade (Fuente: MeSH NLM).

1. Coordinador de Áreas Críticas.

2. Médico intensivista.

a. Hospital Alberto L. Barton Thompson. Callao, Perú. 


\section{INTRODUCCIÓN}

El pericardio normal es un saco de doble pared que contiene el corazón y las raíces de los grandes vasos (1). Contribuye al acoplamiento diastólico entre los dos ventrículos $^{(2,3)}$. Entre el pericardio parietal y visceral, hay una cavidad pericárdica llena de $10-50$ cc de fluido, un ultrafiltrado del plasma producido por el pericardio visceral ${ }^{(1)}$. La acumulación de líquido o sangre en esta cavidad se denomina derrame pericárdico ${ }^{(3)}$.

El derrame pericárdico se puede desarrollar en cualquier enfermedad pericárdica, incluyendo pericarditis y varios trastornos sistémicos, tales como tumores malignos, tuberculosis pulmonar, insuficiencia renal crónica, enfermedades de la tiroides, y enfermedades autoinmunes ${ }^{(1)}$.

La presión pericárdica está determinada por la interrelación entre el volumen de la efusión y la complianza del pericardio. Cuando se acumula líquido en el espacio pericárdico la presión en dicho espacio se incrementa ${ }^{(4)}$.

El taponamiento pericárdico ocurre cuando la presión en el espacio pericárdico excede a la presión en las cámaras cardiacas. Cuando esto sucede, el llenado cardiaco se deteriora. Si es de suficiente severidad, puede producir shock $^{(5)}$.

La ecocardiografía transtorácica es la herramienta más importante para el diagnóstico, clasificación, realización de pericardiocentesis, y seguimiento del derrame pericárdico $^{(1)}$.

La ecografía es muy útil para identificar el derrame pericárdico así como signos sugerentes de taponamiento cardiaco. Con la ecografía bidimensional, el líquido pericárdico aparece como un espacio relativamente libre de eco adyacente al corazón. Dependiendo del tamaño del derrame, éste puede limitarse a un área pequeña dependiente justo posterior a la pared inferolateral del ventrículo izquierdo (VI) en el eje paraesternal. Hay que tener en cuenta que el derrame pericárdico identificado durante la diástole es considerado anormal, independientemente de su volumen y localización ${ }^{(5)}$.

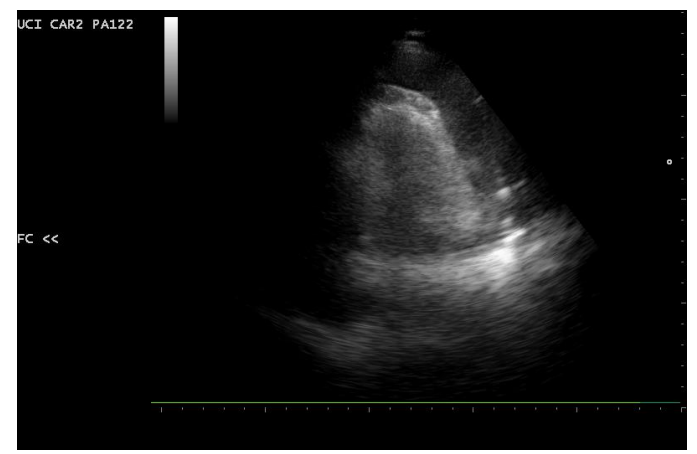

Figura 1. Ecografía cardiaca. Vista apical. Se aprecia importante derrame pericárdico alrededor del VI

\section{CASO CLÍNICO}

Paciente mujer de 50 años con antecedentes de haber sido operada de neoplasia de mama derecha 4 años antes, recibió quimioterapia post cirugía y en tratamiento con anastrozol.

Acude a emergencia por tiempo de enfermedad de un mes caracterizado por disnea progresiva hasta hacerse de reposo y tos. Ingresa inicialmente a medicina interna, pero luego pasa a la Unidad de Cuidados Intensivos (UCI) debido a mal patrón ventilatorio con uso de musculatura accesoria. Ingresa despierta, lúcida, taquipneica, PA: 130/80 mmHg, FC: 95-105 lpm, FR: 30-35/min, Sat.02: $90 \%$ (con mascarilla reservorio), $\mathrm{T}^{\circ}: 37.5^{\circ}$, en posición semisentada. A la auscultación presenta abundantes crépitos bibasales, subcrepitantes en ambos campos pulmonares (ACP) y roncantes difusos, con respiración tóraco-abdominal.

Se maneja inicialmente como una probable neumonía comunitaria. Como parte de la exploración se le realiza ecografía a pie de cama. En la ecografía torácica se aprecia presencia de varias líneas B en ACP sugestivas de edema agudo de pulmón (ver link del video: https: / /youtu. be/bX03nes11R0). En la ecografía cardiaca se aprecia presencia de importante derrame pericárdico (Figura 1), con el signo del corazón oscilante (video: https:// youtu.be/rOj2l8brNYQ), vena cava inferior (VCI) de más de $20 \mathrm{~mm}$ (Figura 2) y colapso sistólico de la aurícula derecha (AD) (video: https://youtu.be/LZ1mZDUnAM8). Ante la presencia de signos ecocardiográficos sugestivos de taponamiento cardiaco y el antecedente de neoplasia maligna se envía al hospital de referencia para realización de ventana pericárdica, la cual es realizada con éxito.

La paciente regresa a nuestra $\mathrm{UCl}$ al día siguiente del procedimiento con evidente mejoría clínica y ecográfica (Figura 3 y video: https://youtu.be/cCm1ZBUF4S4). Permanece 4 días más en UCl en donde se retira el drenaje pericárdico dejado durante la intervención quirúrgica (IQ) y pasa luego a medicina interna para continuar manejo, de donde posteriormente es dada de alta del hospital. La biopsia tomada en la IQ presenta pared pericárdica fibrosa,

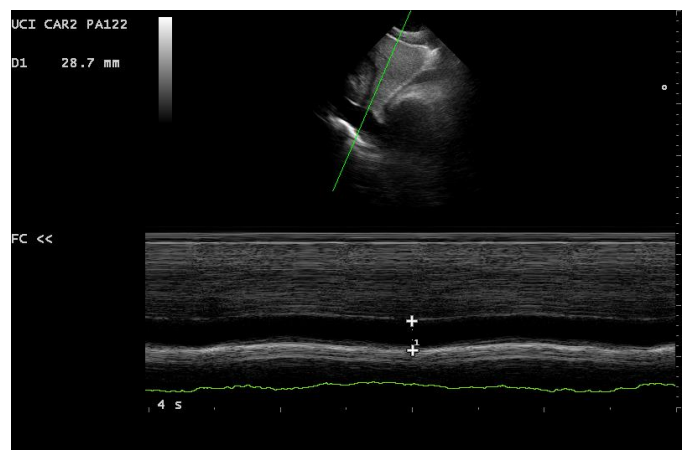

Figura 2. Ecografía cardiaca. Ventana subxifoidea, vista de $\mathrm{VCl}$. Se realiza medición en modo $M$, dando un diámetro de $28,7 \mathrm{~mm}$ 
libre de inflamación y en block cell y PAP se encuentran células mesoteliales con aspecto atípico por lo que es derivada a consultorio de oncología para manejo.

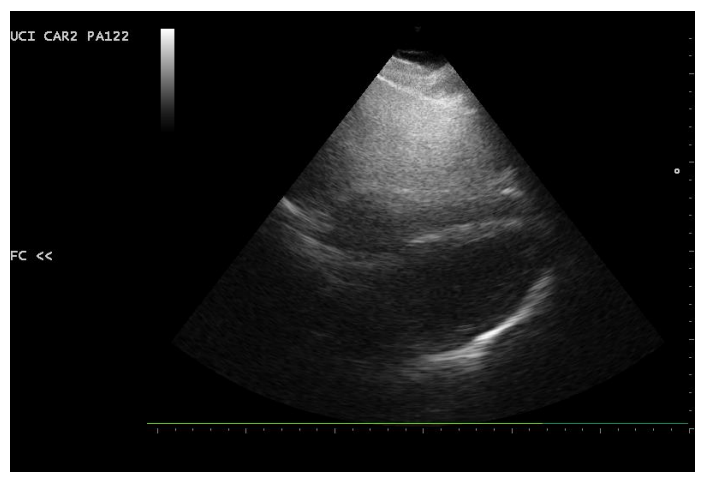

Figura 3. Ecografía cardiaca. Ventana subxifoidea. No se aprecia derrame pericárdico

\section{DISCUSIÓN}

En el taponamiento cardiaco, la interdependencia ventricular es exagerada debido al aumento de la presión intrapericárdica y la limitación del volumen cardíaco global ${ }^{(5)}$. Durante la respiración espontánea, la inspiración produce una reducción desproporcionada del llenado del VI y un aumento de la del ventrículo derecho (VD); lo contrario se produce durante la espiración. Esto se asocia con marcados cambios en el volumen sistólico (VS) durante el ciclo respiratorio y con una reducción inspiratoria $\mathrm{y}$ aumento espiratorio en el VS del $\mathrm{VI}^{\left({ }^{(6)}\right.}$. Esto explica el hallazgo de pulso paradójico en el examen físico de estos pacientes. El efecto predecible del fluido pericárdico en el tamaño de la cámara y la variación respiratoria en el VS del VD y el VS del VI son la base para los hallazgos ecocardiográficos de taponamiento pericárdico ${ }^{(5)}$.

Con el aumento de la presión pericárdica, la aurícula (de baja presión) es la que se afecta primero, seguido por el ventrículo (de presión más alta). La presión pericárdica puede elevarse al grado de causar que las presiones de las cámaras se igualen ${ }^{(5)}$.

La ecocardiografía en modo M y en Doppler 2D es el estándar de oro para el diagnóstico de derrame pericárdico ${ }^{(1)}$.

Los estudios han demostrado un alto grado de sensibilidad y especificidad en la detección de derrame pericárdico usando ultrasonido cardiaco focalizado (FOCUS), que puede ser realizada por médicos no cardiólogos en situaciones de emergencia o al lado de la cama del paciente ${ }^{(7)}$.

Para el intensivista, es suficiente clasificar el tamaño de la colección de fluido cualitativamente como leve, moderado, o severo, dependiendo de la distancia de separación entre el pericardio parietal y visceral: menos de $0,5 \mathrm{~cm}$ es leve, 0,5-2,0 cm moderado, y severo mayor de 2,0 cm. Como ya se dijo, el fluido obedece a las leyes de la gravedad por lo que se localiza en posiciones dependientes en el espacio pericárdico. Un derrame puede parecer pequeño alrededor de la base del corazón, mientras que alrededor del ápex puede parecer grande. La medición se realiza en el punto de separación más grande ${ }^{(5)}$.

Inicialmente, los derrames pequeños son evidentes en la parte posterobasal del VI; sin embargo, cuando el volumen de fluido aumenta, se extiende poco a poco hacia delante, lateralmente, y detrás de la aurícula izquierda $(\mathrm{AI})^{(1)}$.

En presencia de una gran derrame pericárdico, el corazón se vuelve extremadamente móvil dentro del saco pericárdico, un hallazgo que se refiere como "corazón oscilante”. En esa etapa, el taponamiento cardíaco está presente con frecuencia ${ }^{(5)}$.

El líquido pleural se puede confundir con líquido pericárdico. En la vista paraesternal eje largo, un derrame pleural izquierdo aparece como un espacio sin eco posterior al corazón similar a un derrame pericárdico. En esta vista, un derrame pericárdico se ubica anterior a la aorta descendente, mientras que un derrame pleural izquierdo se localiza posterior a la aorta descendente. El líquido que se encuentra exclusivamente posterior a la Al es probable que sea un derrame pleural, debido a la compleja red de reflexiones pericárdicas de esa zona ${ }^{(5)}$.

Si la presión intrapericárdica supera a la presión de la $A D$, se colapsará la pared libre de la $A D$. Esto se ve mejor en la vista apical de cuatro cámaras. La AD normalmente reduce su volumen al final de la diástole (diástole y sístole se refieren ciclo ventricular), de modo que el grado y la duración del colapso de la aurícula son cruciales para determinar si hay compresión de la estructura por un derrame. El colapso sistólico de la $A D$ indica compresión por la efusión pericárdica. La inversión de la pared de la $A D$ durante más de un tercio de la sístole tiene una alta sensibilidad y especificidad para el diagnóstico de taponamiento pericárdico ${ }^{(8-9)}$.

Presiones intrapericárdicas elevadas también pueden causar colapso de la pared del VD ${ }^{(10)}$. El tracto de salida del VD es la más parte compresible del VD, por lo que este colapso paradójico se visualiza mejor en el eje largo paraesternal. Ocurre al inicio de la diástole. Si el colapso se extiende hacia el cuerpo del VD, esto sugiere presiones intrapericárdicas muy elevadas ${ }^{(5)}$. El colapso del VD puede ser atenuado si el VD resiste la compresión debido a hipertrofia o a una enfermedad miocárdica infiltrante ${ }^{(11)}$ o cuando se eleva la presión del VD. Como resultado, el colapso diastólico del VD es más un signo específico más que sensible de taponamiento pericardio ${ }^{(5)}$.

El taponamiento pericárdico genera que la $\mathrm{VCl}$ se dilate sin una variación importante de su tamaño durante el ciclo respiratorio $^{(12)}$.

Normalmente, hay una pequeña variación en el VS entre inspiración y espiración durante una respiración tranquila espontánea. En el lado izquierdo del corazón, el VS cae durante la inspiración, mientras que en el derecho el VS aumenta durante la inspiración. Esta variación respiratoria 
fisiológica se acentúa con el taponamiento pericárdico y es la base para el diagnóstico con Doppler de esta patología ${ }^{(5)}$.

En lugar de medir el VS real, la velocidad de flujo Doppler se puede utilizar como un sustituto del VS. Por ejemplo, una reducción en la velocidad de la onda E mitral mayor del $25 \%$ durante la inspiración (en pacientes con respiración espontánea) es consistente con la fisiología del taponamiento ${ }^{(13,14)}$.

Desde el punto de vista de los intensivistas, hay problemas con el diagnóstico del taponamiento basado en Doppler. Las variaciones respiratorias en el VS y las velocidades del flujo para el diagnóstico de taponamiento pericárdico no han sido validadas para pacientes en ventilación mecánica (15). Estas mediciones sólo deben aplicarse, por tanto, en pacientes que respiran espontáneamente ${ }^{(5)}$.

La ecografía cardiaca es un componente crítico en el diagnóstico ya que identifica la presencia de un derrame pericárdico. Sin embargo, el diagnóstico de taponamiento pericárdico es siempre un diagnóstico clínico y hemodinámico. La presencia o ausencia de colapso de cámara o variaciones respiratorias en las velocidades de flujo es útil, pero no es diagnóstico de la condición. Esto tiene que ser interpretado dentro el contexto clínico ${ }^{(5)}$.

Para el diagnóstico final de taponamiento cardíaco, el médico debe correlacionar los signos ecocardiográficos de taponamiento (colapso de VD, colapso de la AD, alteración respiratoria del flujo mitral y tricuspídeo, y plétora de la $\mathrm{VCl}$ ) con los síntomas y signos de taponamiento clínico (disnea, taquicardia, distensión venosa yugular, pulso paradójico, hipotensión y shock) ${ }^{(16)}$.

En el paciente con falla hemodinámica, la presencia de un derrame pericárdico plantea la posibilidad de taponamiento pericárdico ${ }^{(5)}$.

En conclusión, lo que se hizo fue correlacionar los hallazgos ecográficos de taponamiento cardiaco (corazón oscilante, colapso sistólico de la $\mathrm{AD}$ y $\mathrm{VCl}$ dilatada) con la clínica de la paciente (disnea, taquicardia) y ofrecerle un manejo oportuno antes de que se presenten hipotensión o signos de shock.

\section{REFERENCIAS BIBLIOGRÁFICAS}

1. Jung H. Pericardial Effusion and Pericardiocentesis: Role of Echocardiography. Korean Circ J. 2012; 42(11):725-34.

2. Oh J, Seward J, Tajik A. Pericardial diseases. En: Oh J, Seward J, TajikA editors. The echo manual. 3rd ed. Philadelphia: Lippincott Williams and Wilkins (Wolters Kluwers). 2007; 289-92.

3. Munt B, Moss R, Grewal J. Pericardial disease. En: Otto CM editor. The practice of clinical echocardiography. 4th ed. Philadelphia: Saunders/Elservier. 2011; 565-78.

4. Birks E, Tansley P, Hardy J, George R, Bowler C, Burke M, et al. Left ventricular assist device and drug therapy for the reversal of heart failure. N Engl J Med. 2006; 355(18):1873-84.

5. De Backer D, Cholley B, Slama M, Vieillard-Baron A, Vignon P. Hemodynamic Monitoring Using Echocardiography in the Critically Ill. Springer-Verlag, Berlin: Heidelberg. 2011.
6. Gonzalez M, Basnight M, Appleton C. Experimental cardiac tamponade: a hemodynamic and Doppler echocardiographic reexamination of the relation of right and left heart ejection dynamics to the phase of respiration. J Am Coll Cardiol. 1991; 18(1):243-52.

7. Ceriani $E$, Cogliati C. Update on beside ultrasound diagnosis of pericardial effusion. Intern Emerg Med. 2016; 11(3): 477-80.

8. Gillam L, Guyer D, Gibson T, King M, Marshall J, Weyman A. Hydrodynamic compression of the right atrium: a new echocardiographic sign of cardiac tamponade. Circulation. 1983; 68(2):294-301.

9. Kronzon I, Cohen M, Winer H. Diastolic atrial compression: a sensitive echocardiographic sign of cardiac tamponade. J Am Coll Cardiol. 1983; 2(4):770-5.

10. Armstrong W, Schilt B, Helper D, Dillon J, Feigenbaum $\mathrm{H}$. Diastolic collapse of the right ventricle with cardiac tamponade: an echocardiographic study. Circulation. 1982; 65(7):1491-6.

11. Leimgruber $\mathrm{P}$, Klopfenstein $\mathrm{H}$, Wann L, Brooks $\mathrm{H}$. The hemodynamic derangement associated with right ventricular diastolic collapse in cardiac tamponade: an experimental echocardiographic study. Circulation. 1983; 68(3):612-20.

12. Himelman R, Kircher B, Rockey D, Schiller N. Inferior vena cava plethora with blunted respiratory response: a sensitive echocardiographic sign of cardiac tamponade. J Am Coll Cardiol. 1988; 12(6):1470-7.

13. Appleton C, Hatle L, Popp R. Cardiac tamponade and pericardial effusion: respiratory variation in transvalvular flow velocities studied by Doppler echocardiography. J Am Coll Cardiol. 1988; 11(5):1020-30.

14. Leeman D, Levine M, Come P. Doppler echocardiography in cardiac tamponade: exaggerated respiratory variation in transvalvular blood flow velocity integrals. J Am Coll Cardiol. 1988; 11(3):572-8.

15. Faehnrich J, Noone R, White W, Leone B, Hilton A, Sreeram $G$, et al. Effects of positive-pressure ventilation, pericardial effusion, and cardiac tamponade on respiratory variation in transmitral flow velocities. J Cardiovasc Vasc Anesth. 2003; 17(1):45-50.

16. Sagristà-Sauleda J, Mercé A, Soler-Soler J. Diagnosis and management of pericardial effusion. World J Cardiol.2011; 3(5):135-43.

Fuentes de financiamiento:

Este artículo ha sido financiado por los autores.

Conflictos de interés:

Los autores declaran no tener ningún conflicto de interés.

Correspondencia:

Víctor Pérez Cateriano

Dirección: Calle Carlos Rodrigo 1055, Dpto. 201, Urb. Roma. Lima.

Teléfono: 999779746

Correo electrónico: vpc051@gmail.com

Recibido: 11 de marzo de 2016

Evaluado: 16 de marzo de 2016 Aprobado: 23 de mayo de 2016

(C) La revista. Publicado por Universidad de San Martín de Porres, Perú. (cc) в 1 Licencia de Creative Commons Artículo en acceso abierto bajo términos de Licencia Creative Commons Atribución 4.0 Internacional. (http://creativecommons.org/licenses/by/4.0/) 